\title{
Balbino Marrón y la arquitectura hospitalaria: su prueba de pensado en la Real Academia de Bellas Artes de San Fernando
}

\author{
Balbino Marrón and hospital architecture: his \\ practical project presented at the San Fernando \\ Royal Academy of Fine Arts
}

Alberto FERNÁNDEZ GONZÁLEZ

Universidad de Sevilla

Recibido: 1-III-2015 / Aceptado: 30-IV-2015

Resumen: Balbino Marrón y Ranero fue el arquitecto más destacado del siglo XIX en la ciudad de Sevilla. El trabajo de investigación aporta información inédita localizada en el Archivo de la Academia de San Fernando que aclara las incertidumbres que todavía existían sobre su formación inicial, maestros y primeras influencias. También se analiza pormenorizadamente el proyecto de hospital general para la ciudad hispalense que el joven Marrón presentó en la Academia madrileña como prueba de examen, un relevante documento gráfico que nunca se había estudiado en profundidad, a pesar de que manifiesta una concepción moderna y funcional de la arquitectura hospitalaria.

Palabras clave: Balbino Marrón y Ranero, Arquitectura hospitalaria, Planos y dibujos, Real Academia de Bellas Artes de San Fernando, Sevilla, Siglo XIX.

ABSTRACT: Balbino Marrón y Ranero was the most remarkable architect of 19th century Seville. This research work provides some unpublished information found at the Archives of the Academy of San Fernando which sheds light on the uncertainties that still existed about this architect's initial training, his masters and first influences. It also analyses in detail the project for a General Hospital in Seville which young Marrón presented at the Academy in Madrid as an exam test, a relevant graphic document which had never been studied deeply, despite showing a modern, functional conception of hospital architecture.

Keys words: Balbino Marrón y Ranero, Hospital Architecture, Plans and drawings, San Fernando Royal Academy of Fine Arts, Seville, 19th Century.

Balbino Marrón y Ranero fue el arquitecto más destacado del siglo XIX en la ciudad de Sevilla. Llevó a cabo relevantes intervenciones urbanísticas en los terrenos del Prado de San Sebastián y la margen izquierda del Guadalquivir, y también se 
encargó de la ordenación de la plaza de Armas y la calle Torneo con motivo del establecimiento de las estaciones del ferrocarril. En el interior de la ciudad hispalense, como arquitecto y urbanista, regularizó la plaza del Museo, terminó la fachada del edificio que alberga el actual Museo de Bellas Artes y culminó el proyecto de la plaza Nueva y el frente ochocentista del edificio del Ayuntamiento. Se ocupó igualmente de la alineación de la Alameda de Hércules, la construcción del cementerio de San Fernando y la edificación de los mercados del Perneo y los situados en la calle Feria. La transformación del palacio de San Telmo como residencia de los duques de Montpensier también fue obra suya ${ }^{1}$.

De los primeros años de Balbino Marrón apenas se conocían datos significativos, salvo que era vizcaíno y había nacido en Villaro, hoy Areatza, en 1812. Es más, la información que aporta su título de arquitecto, expedido el 24 de septiembre de 1837 en Madrid, se ha malinterpretado, por la falta de datos concretos, sobre todo, incluyendo a Marrón en las últimas promociones de alumnos que cursaron estudios de arquitectura en la Real Academia de Bellas Artes de San Fernando, o suponiendo incluso que su vinculación con Sevilla se inicia en 1838, año en que se declaró vecino de la ciudad al solicitar su primer destino en Jerez como arquitecto municipal ${ }^{2}$. La documentación

${ }^{1} \mathrm{Al}$ respecto, véanse A. GONZÁLEZ DORADO, Sevilla: centralidad regional y organización interna de su espacio urbano, Madrid, 1975; A. GONZÁLEZ CORDÓN, Vivienda y ciudad: Sevilla 1849-1929, Sevilla, 1985; J. M. SUÁREZ GARMENDIA, Arquitectura y urbanismo en la Sevilla del siglo XIX, Sevilla, 1986, pp. 48-54; ÍDEM, "Balbino Marrón y Ranero", en Diccionario Biográfico Español, vol. XXXII, Madrid, 2009, pp. 708-709; A. POZO Y BARAJAS, Arrabales de Sevilla, morfogénesis y transformación: el arrabal de los Humeros, Sevilla, 1996; J. RODRÍGUEZ BARBERÁN, Los cementerios en la Sevilla contemporánea: análisis histórico artístico, Sevilla, 1996; y V. LLEÓ CAÑAL, La Sevilla de los Montpensier. Segunda corte de España, Sevilla, 1997.

2 J. M. SUÁREZ GARMENDIA, Arquitectura y urbanismo..., pp. 99-104; ÍDEM, "Balbino Marrón...", pp. 708-709. localizada en el Archivo de la Academia de San Fernando, no obstante, es concluyente y además de aclarar algunas incertidumbres biográficas aporta valiosa información sobre la formación inicial del futuro arquitecto municipal y provincial de Sevilla y respecto a sus maestros e influencias ${ }^{3}$.

En mayo de 1837 el joven Balbino Marrón se desplazó desde Sevilla, donde residía, a Madrid con la intención de superar el riguroso examen de la Academia de Bellas Artes y obtener el título de arquitecto ${ }^{4}$. Los aspirantes al título oficial, para ser admitidos en las pruebas, según establecía la normativa vigente en ese momento, que se regulaba por la Real Orden de 28 de agosto de 1816, tenían que acreditar los estudios realizados en la disciplina, si no eran alumnos de la Academia, y certificar un mínimo de dos años de práctica al lado de un académico o arquitecto. También debían presentar el diseño de un edificio de primer orden, ejercicio que se denominaba prueba de pensado y constaba de dos partes: los planos del proyecto, en planta, sección y alzado; y el informe práctico facultativo, que comprendía ubicación, método constructivo y presupuesto económico. Si el aspirante obtenía el beneplácito de la Comisión de Arquitectura, podría presentarse a la segunda fase de la oposición, la prueba de repente, que se celebraba en una sala de la Academia donde se aislaba al aspirante a arquitecto durante un tiempo fijo no supe-

\footnotetext{
${ }^{3}$ Archivo de la Real Academia de Bellas Artes de San Fernando (en adelante ARABASF), Comisión de Arquitectura, Expediente 2-4-6, s. fol.

${ }^{4}$ La madrileña de San Fernando y la valenciana de San Carlos eran las únicas academias que en ese momento tenían la potestad de emitir los títulos oficiales de arquitecto y maestro de obras (M. R. SANTAMARÍA ALMOLDA, “Bases documentales para el estudio de la teoría arquitectónica (1814-1858) en la Real Academia de Bellas Artes de San Fernando", Espacio, Tiempo y Forma, Serie VII, no 9, 1996, p. 220. En Sevilla, la Escuela de las Tres Nobles Artes fundada por Carlos III en 1771 alcanzó el rango de academia en 1843 y la consideración de primera clase en 1849. Al respecto, véase A. MURO OREJÓN, Apuntes para la historia de la Academia de Bellas Artes de Sevilla, Sevilla, 1961, pp. 67 y 259.
} 
rior a quince horas a fin de que delinease un proyecto escogido de entre tres temas salidos al azar del Libro de asuntos para arquitectos. Además de las pruebas de pensado y de repente, los opositores tenían que superar un examen oral ante un tribunal académico, en el transcurso del cual se cotejaban los planos hechos en ambas fases y se preguntaba al concursante sobre ellos y acerca de las materias propias de la profesión, tanto teóricas como prácticas. Si el dictamen de los académicos, establecido mediante votación secreta, era favorable, el aspirante obtenía el título de arquitecto ${ }^{5}$.

El expediente completo de la oposición realizada por Balbino Marrón se conserva en el Archivo de la Academia. El documento se ajusta a la normativa legal, como era preceptivo, e incluye la partida de nacimiento y varios certificados relativos a los estudios y practicas realizadas por el aspirante a arquitecto. Marrón, tal y como constata Pedro José de Madariaga, sacerdote de la iglesia parroquial de San Bartolomé de la villa de Villaro, era hijo legítimo de León Marrón, natural de Bilbao, y Lorenza Ranero, que había nacido en Villanueva de Presa, pueblo del municipio de Carranza, en Vizcaya, y vino al mundo en el referido pueblo vizcaíno de Villaro, hoy Areatza, "el 23 de febrero de 1812, a la una y media poco más o menos de la mañana" ${ }^{\prime \prime}$.

La documentación, por desgracia, no aclara el motivo ni la fecha concreta de su traslado a Sevilla porque esa información, al fin y al cabo, era irrelevante para el examen, pero incluye una certificación firmada por Melchor Cano en la ciudad de la Giralda el

${ }^{5}$ Sobre esta cuestión, véanse J. E. GARCÍA MELE$\mathrm{RO}$, “Arquitectura y Burocracia: el proceso del proyecto en la Comisión de Arquitectura de la Academia (17861808)", Espacio, Tiempo y Forma, Serie VII, no 4, 1991, pp. 283-348; ÍDEM, "El debate académico sobre los exámenes para las distintas profesiones de la Arquitectura (1781-1783): El arquitecto según Juan de Villanueva", Ibídem, no 6, 1993, pp. 325-377 y M. R. SANTAMARÍA ALMOLDA, Op. cit., pp. 219-225.

${ }^{6}$ ARABASF, Comisión de Arquitectura, Expediente 2-4-6, s. fol.
5 de mayo de 1837, en calidad de "Arquitecto Académico de Mérito de las Academias Nacionales de San Fernando y de San Carlos, y Arquitecto mayor de Sevilla, y Director de Arquitectura de la Escuela de Nobles Artes hispalense", que acredita que Balbino Marrón "ha estado cuatro años estudiando la arquitectura, y asistiendo a las obras que tuvo el arquitecto Don Salustiano Ardanaz, y después de la muerte de este ha continuado concurriendo a la clase de arquitectura de la Escuela de Nobles Artes con el mayor aprovechamiento y aplicación, asistiendo también a las obras que han estado bajo mi dirección; hallándose bastante impuesto en la práctica de construcción"7.

Marrón, por tanto, no se formó en Madrid, en la Academia de San Fernando, como se suponía, sino en la Escuela de las Tres Nobles Artes de Sevilla, bajo la dirección, eso sí, de dos arquitectos académicos: Salustiano Ardanaz y Melchor Cano. Al igual que Marrón, Ardanaz era de origen vasco ${ }^{8} \mathrm{y}$ en Sevilla estuvo vinculado a un grupo de intelectuales y científicos -entre los que se encontraba Cano- que se reunía en una casa de la calle Cervantes, donde celebraban conferencias sobre cuestiones matemáticas, de arquitectura, geodesia y otras ramas de las ciencias exactas ${ }^{9}$. Aunque no era profesor en la referida escuela artística, Salustiano Ardanaz tuteló al joven Marrón en la práctica de la profesión a raíz, quizá, de la suspensión temporal de las clases por motivos económi$\cos ^{10 .}$ El madrileño Melchor Cano fue el primer arquitecto aprobado por la Academia que desempeñó el cargo de arquitecto ma-

\footnotetext{
${ }^{7}$ Ibídem.

${ }^{8}$ Fue aprobado maestro arquitecto el 18 de febrero de 1827 (J. LABORDA YNEVA, El proyecto de arquitectura en la Real Academia de Bellas Artes de San Fernando. Las pruebas de examen de los aspirantes vascos. 1776-1855, Vitoria-Gasteiz, 2011, p. 96).

${ }^{9}$ M. ÁLVAREZ BENAVIDES, Explicación del plano de Sevilla, Sevilla, 1868, tom. II, pp. 120-121.

${ }^{10}$ Sobre las dificultades económicas por las que atravesó la institución en el periodo 1831-1835, véase A.
} MURO OREJÓN, Op. cit., p. 258. 
yor de la ciudad de Sevilla ${ }^{11}$. Desde 1827 era el responsable de las enseñanzas de "Geometría práctica y descriptiva" en la Escuela de las Tres Nobles Artes, y a partir de 1829 y hasta su fallecimiento en 1842 ejerció la dirección de su sección de arquitectura ${ }^{12}$.

Otro documento importante que forma parte del expediente y sirve para completar el perfil formativo de nuestro arquitecto lo constituye la carta que el secretario de la Comisión de Arquitectura envía al director de la Academia de San Fernando para informarle de que el aspirante Balbino Marrón había obtenido "la totalidad de sufragios para su admisión a los ejercicios referidos de prueba y examen para graduarse de arquitecto", pues en ella consta que el joven Marrón se inició en el "estudio de la arquitectura en Bilbao al lado del señor Belauzarán" y que había continuado su formación después, "por más de seis años, bajo la dirección de Salustiano Ardanaz, y a su fallecimiento, de Melchor Cano"13. Es decir, el primer maestro de Marrón, en Bilbao, antes de trasladarse a Sevilla, fue el arquitecto neoclásico Juan Bautista Belaunzarán ${ }^{14}$. La edad a la que co-

\footnotetext{
${ }^{11}$ Toma posesión de su cargo en Sevilla el 16 de enero de 1826 (J. M. SUÁREZ GARMENDIA, Arquitectura y urbanismo..., p. 49).

${ }^{12}$ A. MURO OREJÓN, Op. cit., pp. 48 y 50.

${ }^{13}$ ARABASF, Comisión de Arquitectura, Expediente 2-4-6, s. fol.

${ }^{14}$ Su obra más importante fue el cementerio bilbaíno de Mallona, un trabajo de 1823 que sigue los parámetros higienistas y clásicos (sobre este arquitecto, véanse K. M. BARAÑANO, J. GONZÁLEZ DE DURANA y J. JUARISTI, Arte en el País Vasco, Madrid, 1987, p. 168 y J. A. BARRIO LOZO, "Los cementerios neoclásicos porticados del País Vasco. El caso de Vizcaya", en Una arquitectura para la muerte, Sevilla, 1993, pp. 291-295; ÍDEM, "El urbanismo y la arquitectura neoclásica en el País Vasco", Ondare, no 21, 2002, pp. 15-45). A la luz de esta referencia documental que hace de Belaunzarán el primer maestro de Marrón resulta obligado comparar el cementerio bilbaíno de Mallona y el sevillano de San Fernando. Aunque las influencias concretas son muy difíciles de precisar, no cabe duda de que el proyecto que elaboró Balbino Marrón en junio de 1851 para el cementerio hispalense, cuando ya era un arquitecto experimentado, nada tiene que ver con la concepción
}

menzó sus estudios en la capital de Vizcaya no se puede establecer con certeza pero las referencias documentales permiten fechar su llegada a Sevilla hacia el año 1831, cuando Marrón tenía unos 18 o 19 años. Una posible recomendación de Belaunzarán al también vasco Ardanaz, que trabajaba como arquitecto en la ciudad de la Giralda, no se debe descartar.

También forma parte del expediente que se conserva en el Archivo de la Real Academia de Bellas Artes de San Fernando un certificado de buena conducta que firmó José Ramírez Cruzado, otro sacerdote, titular en este caso de la parroquia de San Andrés de la ciudad de Sevilla. En este documento figuran un par de datos interesantes sobre el joven Marrón y Ranero: que "ha observado continuamente buena vida y costumbres sin tacha alguna en su conducta moral y política", y que había vivido "en la feligresía cuatro años continuos hasta el mes de mayo de 1837 en que pasó a la villa y corte de Madrid"15. Al margen de la primera información, que se ajusta a lo esperado en un futuro arquitecto, se constata que en el periodo 1833-1837 vivía Marrón en la parroquia de San Andrés, muy cerca del mercado de la Encarnación que por esas fechas construían sus maestros Ardanaz y Cano y con los que seguramente colaborón ${ }^{16}$.

funeraria de Juan Bautista Belaunzarán. Marrón, de hecho, fue muy crítico con las formas de edificación neoclásicas porticadas y las sepulturas de inhumación y nichos bajo galerías, que considera carentes de higiene, y defiende mausoleos y sepulturas subterráneas como elementos básicos (F. J. RODRÍGUEZ BARBERÁN, Los cementerios en la Sevilla contemporánea: análisis histórico y artístico (1800-1950), Sevilla, 1996, p. 107).

15 ARABASF, Comisión de Arquitectura, Expediente 2-4-6, s. fol. Se entiende, claro está, que marchó a Madrid para realizar las pruebas preceptivas y obtener el título de arquitecto.

${ }^{16}$ Sobre su historia constructiva, véase A. FERNÁNDEZ GONZÁLEZ, "Arquitectura y urbanismo en la Sevilla ochocentista: la plaza-mercado de la Encarnación", Anuario del Departamento de Historia y Teoría del Arte, $\mathrm{n}^{\circ}$ 21, 2009, pp. 205-222. 
En mayo de 1837, por tanto, Balbino Marrón se encontraba en Madrid preparando su prueba de pensado, ejercicio de gran complejidad que servía para constatar los conocimientos teóricos y técnicos de los aspirantes a arquitecto en los métodos y materiales constructivos y en cuanto a tipologías $^{17}$. Tres meses más tarde, el 12 de agosto de 1837 concretamente, presenta Marrón, en la secretaría de la Academia, la solicitud para ser admitido en la primera fase de la oposición, que acompaña de los certificados y ejercicios requeridos.

El proyecto escogido por Balbino Marrón fue el diseño de un hospital general con capacidad para setecientas plazas de ambos sexos, conjunto edilicio que, según explica en su informe facultativo, sitúa en Sevilla, "por reunir en un solo edificio los varios que con el mismo objeto existen dentro de la referida ciudad"18, pero fuera de la muralla, en la periferia de la localidad, por motivos de higiene pública ${ }^{19}$. Los tres

${ }^{17}$ La mayor parte de las pruebas conservadas en la Academia de San Fernando fechadas en las primeras décadas del ochocientos se refieren a edificios públicos de primer orden, como teatros, hospitales, ayuntamientos, bibliotecas, templos, cuarteles, cementerios o museos. Al respecto, véase $\mathrm{M}$. R. SANTAMARÍA ALMOLDA, Op. cit., p. 221.

18 "Excelentísimo Señor: Siendo indispensable según exigen las Reales Ordenanzas vigentes la presentación de planos geométricos de un edificio cualquiera de primer orden, para obtener de la Academia Nacional el título de arquitecto, y quedando a mi arbitrio su elección, forma y dimensiones, he dispuesto los de un Hospital General para una ciudad capital de provincia capaz de contener con la debida comodidad setecientas personas de ambos sexos con sus respectivas dependencias. La ciudad de Sevilla, capital de la provincia me ha parecido el punto más adecuado para su colocación..." (ARABASF, Comisión de Arquitectura, Expediente 2-4-6, informe facultativo, fols. 1 r. y v.).

${ }^{19}$ La progresiva necesidad de más higiene social y un mayor espacio para las instalaciones sanitarias impondrá un alejamiento de los hospitales del centro de la ciudad burguesa del siglo XIX. Esta norma constructiva ya la defendían B. BAILS, Elementos de Matemática, T. IX, Madrid, 1783, pp. 855, 857 y F. A. VALZANIA, Instituciones de arquitectura, Madrid, 1792, p. 64. También Melchor Cano, maestro de Marrón en el momento planos que constituyen la prueba de pensado realizada por el joven Marrón han sido publicados recientemente por José Laborda Yneva en una obra muy instructiva y necesaria que recopila las pruebas de examen de los aspirantes vascos comprendidas entre los años 1776 y 1855 que se conservan en el Archivo de la Academia de San Fernando $^{20}$. Laborda, eso sí, dado el enorme volumen de la documentación gráfica que ha manejado, que alcanza 848 planos correspondientes a 381 proyectos, sólo ha podido dedicar breves fichas de análisis a los diseños de los arquitectos vascos, y los planos trazados por Marrón, desde luego, merecen un estudio más pormenorizado que tenga en cuenta las características del tipo hospitalario en su contexto históricoartístico y sus posibles influencias y modelos de inspiración.

El primero de los tres documentos gráficos proyectados por el joven Balbino Marrón al que voy a referirme es el correspondiente a la fachada del hospital general ${ }^{21}$ (Fig. 1). Se trata, como evidencia el alzado (Fachada principal) y el propio Marrón explica en su informe facultativo ${ }^{22}$, de un edificio sobrio de dos pisos que sigue normas clasicistas en su concepción -no podía ser de otra manera, por supuesto, ya que iba a ser examinado por la Comisión de Arquitectura de la Academia de San Fernando- y presenta una sección central con doble avance sobre el plano principal de fachada hacia una escalinata apenas distinguible en el dibujo,

en que éste presentó su prueba de pensado en Madrid, estaba a favor de la ubicación periférica de los hospitales, tal como evidencia el discurso que pronunció en la Real Academia de San Fernando con motivo de su nombramiento como académico de mérito. Sobre esta cuestión, véase M. A. ANTIGÜEDAD DEL CASTILLOOLIVARES, "El arquitecto Melchor Cano y la teoría de la ciudad", Espacio, Tiempo y Forma, Serie VII, no 3, 1990, p. 424.

${ }^{20} \mathrm{~J}$. LABORDA YNEVA, Op. cit., pp. 312-313.

${ }^{21}$ ARABASF, Gabinete de Dibujos, A-2505.

${ }^{22}$ Ibídem, Comisión de Arquitectura, Expediente 2-4-6, informe facultativo, fols. 4 r. $-4 \mathrm{v}$. 


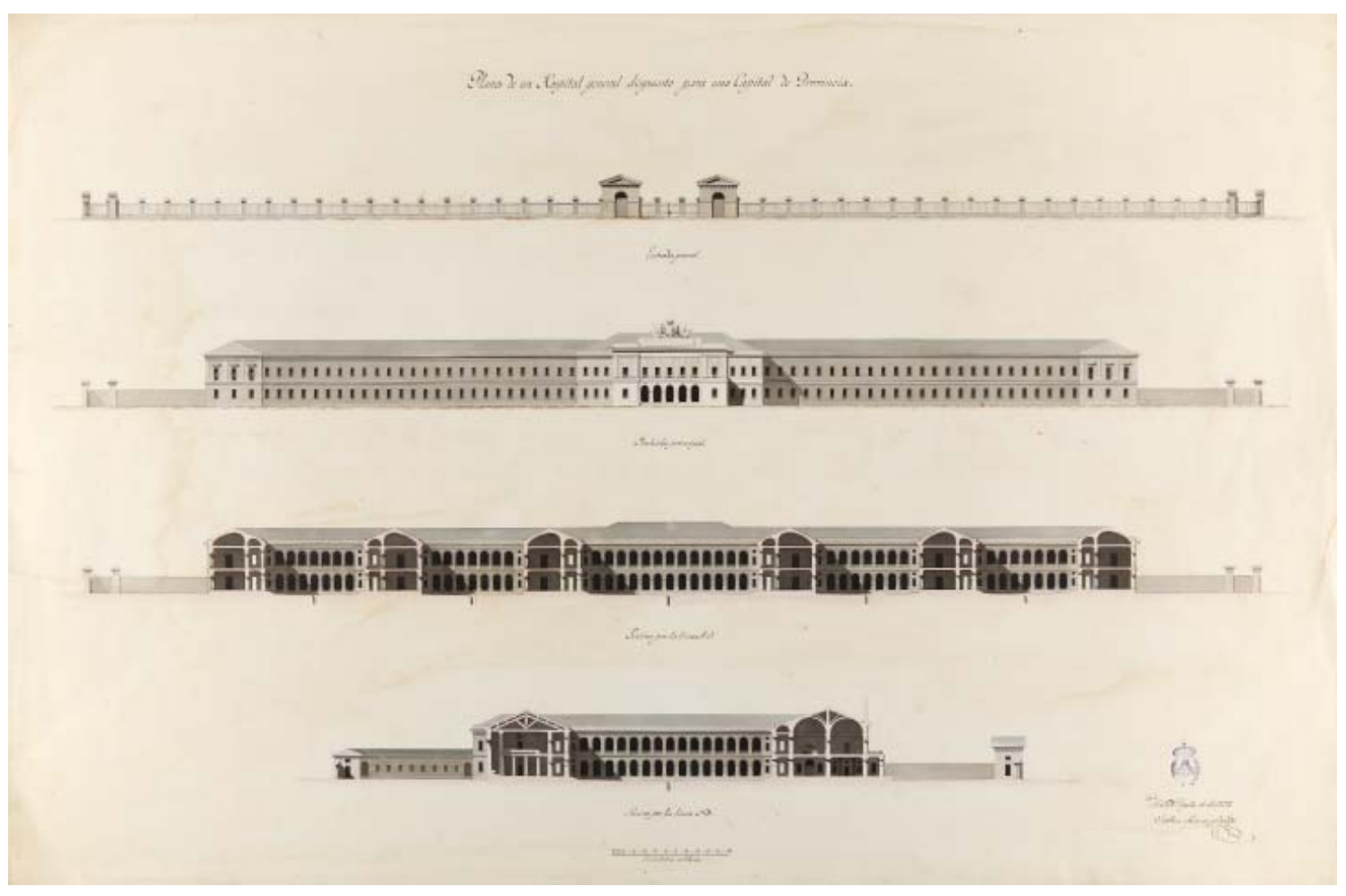

- Fig. 1. Hospital general: alzado y sección. Proyecto de Balbino Marrón, 1837. Archivo de la Real Academia de Bellas Artes de San Fernando, Gabinete de dibujos, A-2505.

aunque mencionada por el futuro arquitecto hispalense en su informe.

El cuerpo medio del imafronte se organiza en pórtico bajo estructurado por cinco arcos semicirculares, amplia balaustrada que cierra el balcón del piso superior y frontón recto coronado por un copete armero que integra escudo y triunfos, triple combinación de elementos que define la fachada principal del antiguo Consistorio de San Sebastián, obra de Silvestre Pé$\mathrm{rez}^{23}$, aunque la balaustrada del piso alto y el remate en copete remiten a fórmulas barrocas. Salvo esta sección central, que protagoniza la arquitectura, el aspecto exterior del edificio es puro módulo, pues el ritmo austero y repetitivo del diseño sólo se altera mínimamente en las tres últimas ventanas altas de cada flanco, que presentan molduras ornamentales y un mayor desarrollo. El ordenamiento reiterativo de

${ }^{23} \mathrm{Al}$ respecto, véase C. SAMBRICIO, La arquitectura española de la Ilustración, Madrid, 1986, pp. 227 y 393. sus vanos podría estar inspirado en el Hospital General de Madrid, edificio diseñado por José de Hermosilla y Francesco Sabatini y nunca completado, que sufrió un largo y complejo proceso constructivo. Con todo, el aspecto de este importante establecimiento sanitario en 1837, cuando Marrón residía en Madrid, no sería muy diferente del plasmado por León Gil de Palacio en su conocida maqueta de Madrid ${ }^{24}$.

El corte longitudinal (Sección por la línea $A B$ ) describe la articulación de los seis pabellones de enfermos proyectados, tres en el flanco izquierdo de las mujeres y otros tantos en el lado opuesto de los hombres. En todos ellos se distinguen las respectivas salas de enfermos bajas y altas, el desván y las galerías de comunicación que se disponen asimismo en ambas plantas. También se detalla el aspecto de los cinco patios que separan los pabellones y las respectivas ga-

\footnotetext{
${ }^{24}$ La reproducción del hospital realizada por Gil de Palacio se puede consultar en Ibídem, p. 207.
} 
lerías de arcos, impostados en el piso bajo y sencillos en el principal. La capilla, de dos alturas y planta cuadrangular, se localiza justo detrás del largo corredor central. Es una pieza claramente funcional que se integra en el hospital y no presenta cúpula ni signos exteriores que la identifiquen como $\operatorname{tal}^{25}$.

El corte transversal (Sección por la línea $C D$ ) manifiesta el fondo del edificio y describe, de izquierda a derecha, la configuración edilicia de varias estancias del hospital: depósito de muertos, capilla y piezas contiguas, patio principal, galería, zaguán, pórtico y cuerpo de guardia ${ }^{26}$.

En este primer plano también figuran dibujados el muro que circunvala el área hospitalaria (Entrada general) y un amplio espacio de transición hacia el edificio donde Marrón tenía previsto instalar jardines y $a^{\prime r b o l e s}{ }^{27}$. En la fachada principal, en vez de muro de cierre, se colocaría el enrejado de hierro con pedestales que aparece delineado con dos puestos de guardia protegiendo la entrada al recinto ${ }^{28}$. Esta solución de verjas y pilares la volverá a emplear Balbino Marrón en su proyecto de 1851 para el cementerio de San Fernando ${ }^{29}$.

Otro plano que custodia la Academia $^{30}$ (Fig. 2) describe el primer piso (Planta baja) del complejo hospitalario imaginado por el joven Marrón. El dibujo comprende el edificio y su espacio adyacente $\left(n^{\circ}-3\right)$ reservado, como se explicó, para el recreo

${ }^{25}$ ARABASF, Comisión de Arquitectura, Expediente 2-4-6, informe facultativo, fol. $5 \mathrm{r}$.

${ }^{26}$ Ibídem, fols. 5 r. $-5 \mathrm{v}$.

27 "En el espacio comprendido entre este cerrado y el del edificio se hallan jardines y arbolados que al mismo tiempo de ser un adorno agradable sirven de salubridad" (Ibídem, fol. 3 r.).

${ }^{28}$ Ibídem, fol. 2 v.

${ }^{29}$ Véase al respecto el dibujo arquitectónico publicado por F. J. RODRÍGUEZ BARBERÁN, "El plano del Cementerio de San Fernando, obra de Balbino Marrón y Ranero", Archivo Hispalense, nº 221, 1989, pp. 165-183.

${ }^{30}$ ARABASF, Gabinete de Dibujos, A-2503. de los usuarios. Se aprecia el amplio muro delineado en el alzado, que define ahora todo el perímetro del terreno salvo el frente principal, donde se proyectaba colocar el referido enrejado de hierro con pedestales. Justo en el centro de esta reja aparecen dibujadas la entrada general (no 1$)$ y dos estancias reservadas al cuerpo de guardia ( $\mathrm{n}^{\mathrm{o}}$ 2).

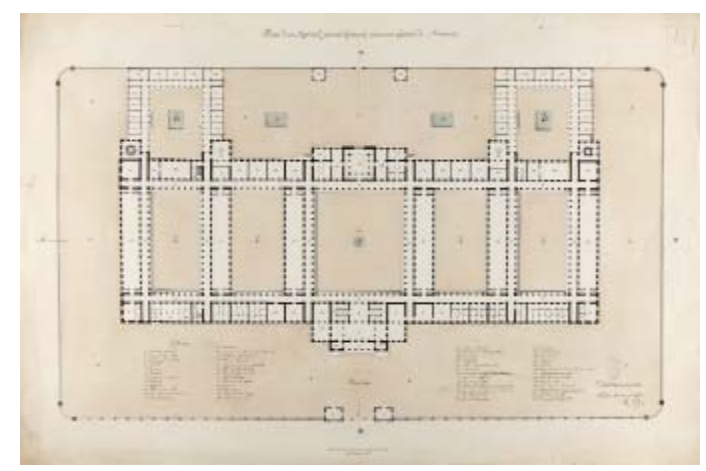

- Fig. 2. Hospital general: planta baja. Proyecto de Balbino Marrón, 1837. Archivo de la Real Academia de Bellas Artes de San Fernando, Gabinete de dibujos, A-2503.

La estructuración planimétrica del hospital es definida por Marrón mediante un diseño en peine que enlaza la crujía principal y la posterior mediante cuerpos transversales a los que dota de salas para enfermos, solución que favorece la relación axial entre estas dos crujías longitudinales. El patio principal, de mayor tamaño, lo sitúa en el centro del edificio para separar el sector masculino, localizado a la derecha, del femenino, ubicado a la izquierda. En su eje medio dispone una fuente "cuyas aguas, juntamente con las de otras dos, de las que haré mención más adelante, surten a dos estanques, desde los cuales pasando por los depósitos de los comunes caminan hacia el río" ${ }^{\prime 1}$.

La crujía correspondiente a la fachada principal, "cuya longitud es de 900 pies castellanos", está muy compartimentada, a fin de albergar un buen número de servi-

\footnotetext{
${ }^{31}$ ARABASF, Comisión de Arquitectura, Expediente
} 2-4-6, informe facultativo, fol. $1 \mathrm{v}$. 


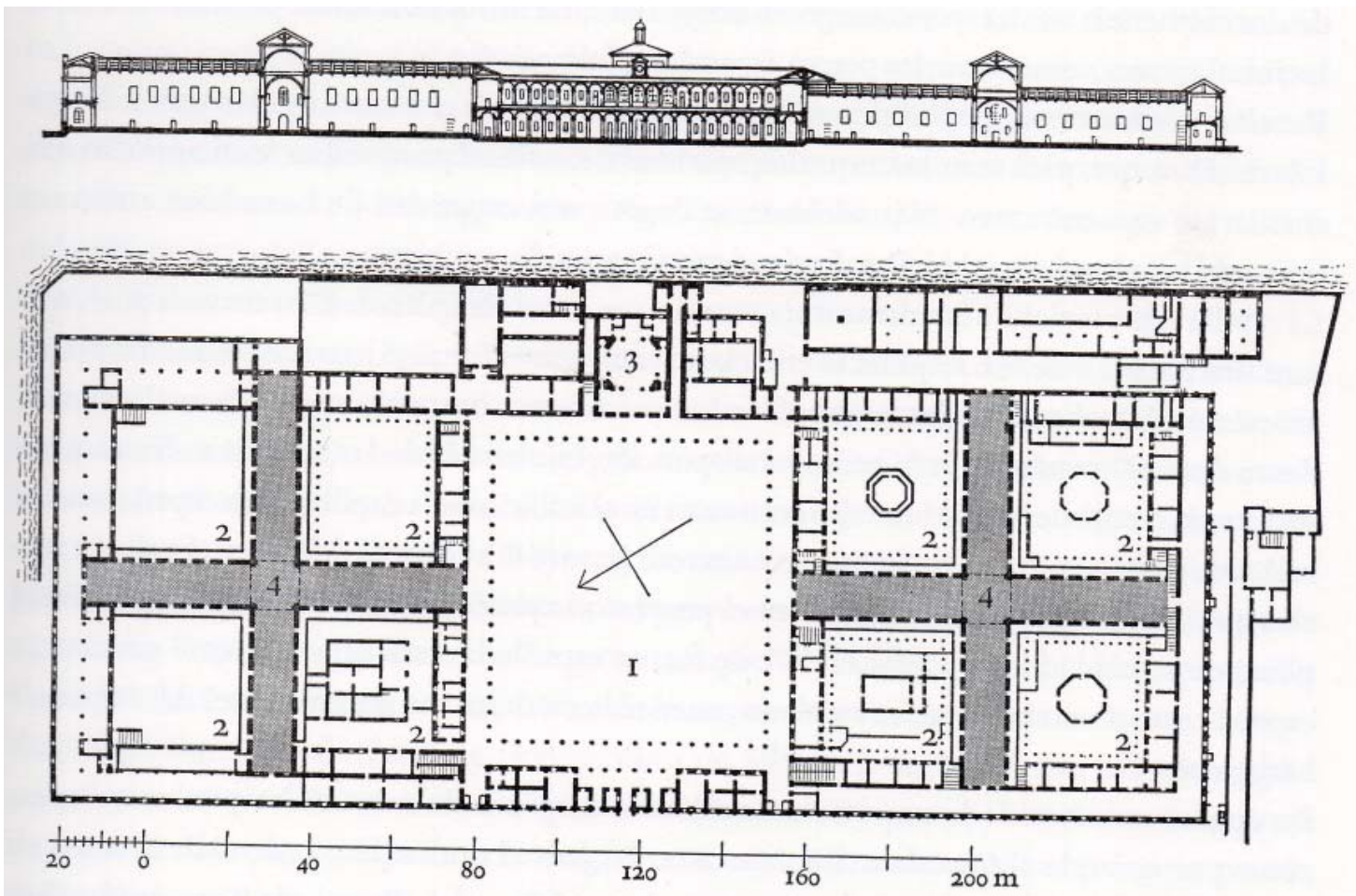

- Fig. 3. Hospital Mayor de Milán. Planta según Tollet. Publicada en D. Leistikow, Edificios hospitalarios en Europa durante diez siglos, Frankfurt am Main, 1967.

cios hospitalarios. En ella ubica Marrón piezas importantes: en su sección central sitúa la escalinata de acceso ( $\mathrm{n}^{\mathrm{o}}$ 1), flanqueada por sendas porterías (no 42 ), que da paso a un zaguán ( $\left.\mathrm{n}^{\circ} 5\right)$ y a un espacio de tránsito $\left(n^{\circ} 6\right)$ que permite el acceso a una escalera de doble tiro (n⿳⺈ 7). El zaguán (n⿳⺈ 5), a su vez, comunica con la secretaría del hospital (no 41), que ocupa dos estancias del lado izquierdo, y la botica (no 9), también de dos piezas, pero emplazada a la derecha. La lógica distributiva se mantiene en los cuartos contiguos de cada flanco: a la izquierda, definiendo un sector administrativo, se localiza la contaduría (no 40), el archivo (no 39) y las habitaciones del secretario $\left(n^{\circ}\right.$ 38) y el contador (no 37) y otra sala sin asignación, tal vez reservada para el personal auxiliar. A la derecha, al lado de la botica (n⿳⺈ 9), se ubican dos dependencias proyectadas como almacenes de botica ( $\mathrm{n}^{\mathrm{o}}$ 10), las habitaciones del boticario ( $\left.\mathrm{n}^{\circ} 11\right)$ y el médico ( $\left.\mathrm{n}^{\mathrm{o}} 12\right)$, y las que iban a ocupar los mozos de botica ( $\mathrm{n}^{\circ}$ 36). A continuación, y en ambos flancos de la crujía, dibuja Marrón sendas escaleras de servicio ( $\left.n^{-0} 13\right)$ que permiten el acceso directo al piso alto y al desván ${ }^{32}$. Hacia el extremo derecho coloca las denominadas salas de enfermos de distinción (n⿳ 35), localización que repite en el sector opuesto, donde sitúa los cuartos de las mujeres distinguidas ( $\left.\mathrm{n}^{\mathrm{o}} 14\right)$. Y justo en los ángulos, formando bloques cuadrangulares, uno en cada extremo de la crujía, aparecen delineadas tres dependencias interconectadas: los denominados gabinetes del aparato (no 51 ), que ocupan dos estancias, y la sala de operaciones ( $\left.n^{\mathrm{o}} 15\right)$, mucho más amplia.

El frente posterior presenta una secuencia horizontal equiparable a la fachada principal. Buscando una clara simetría con la sección central correspondiente a la crujía del frente principal, traza Marrón un espacio similar en la crujía posterior, pero ahora, en vez de pórtico, zaguán y área administrativa, sitúa la capilla ${ }^{33}$ ( $\left.n^{\circ} 29\right)$, en eje

\footnotetext{
${ }^{32}$ Ibídem, fol. 2 r.

${ }^{33}$ Inercia estructural que remite al origen plurifuncional del hospital medieval. Al respecto, véase N. PE-
} 
con el patio principal y la entrada general al complejo sanitario, disposición, por cierto, que repite el esquema del Hospital Mayor de Milán (Fig. 3). A ambos lados de esta pieza, y flanqueadas, a su vez, por varias habitaciones, dos de ellas reservadas para los capellanes ( $\left.\mathrm{n}^{\circ} 27\right)$, se localizan un par de sacristías $^{34}$ ( $\left.\mathrm{n}^{\mathrm{o}} 28\right)$. A continuación organiza Marrón dos bloques idénticos que sitúa contiguos al área religiosa, donde ubica los cuartos de la ropa ( $\left.\mathrm{n}^{\mathrm{0}} 24\right)$, salas de dementes (no 26) y sarnosos ( $\left.\mathrm{n}^{\mathrm{o}} 25\right)$, las piezas identificadas como aparadores ( $\mathrm{n}^{-}$23) y otras estancias de almacenaje de ropa ( $\left.\mathrm{n}^{\mathrm{o}} 24\right)$. Los extremos de esta crujía posterior son ocupados por dependencias diferentes, según el lado: a la izquierda, en el sector reservado para las mujeres, sitúa una escalera ( $\left.\mathrm{n}^{-0} 13\right)$ que comunica con el piso alto y desván, la sala de parturientas ( $\mathrm{n}^{-}$30) y el cuarto de labor ( $\left.\mathrm{n}^{\mathrm{0}} 34\right)$. En el lado derecho correspondiente a los hombres, tras otro acceso de servicio a la planta superior ( $\left.\mathrm{n}^{\mathrm{o}} 13\right)$, se localiza una zona de baños de vapor ( $\left.n^{-} 21\right)$ y sanitarios $\left(\mathrm{n}^{\mathrm{o}} 20\right)$.

La crujía principal y la posterior son enlazadas perpendicularmente mediante seis funcionales y rectangulares pabellones de hospitalización -los tres de la izquierda acogen pacientes femeninos ( $\mathrm{n}^{\mathrm{o}}$ 33) y los demás, situados en el sector derecho, masculinos ( $\mathrm{n}^{\mathrm{o}}$ 16)- que se conciben como núcleos sanitarios incrustados dentro del edificio. A los pabellones se accede mediante galerías de comunicación ${ }^{35}$ ( $\left.n^{\mathrm{o}} 8\right)$ que organizan las circulaciones principales del hospital estableciendo coherentes ejes transversales $\mathrm{y}$ longitudinales que evitan interferencias en el servicio. La eficiente ventilación de las salas

VSNER, Historia de las tipologías arquitectónicas, Barcelona, 1979 , pp. $165-166$.

${ }^{34}$ Como recomendaba F. A. VALZANIA, Op. cit., p. 65.

${ }^{35}$ El diseño de Marrón mantiene la tradición hospitalaria renacentista de patios con galerías de columnas. Sobre la estructura del modelo de hospital propuesto por Filarete, véase D. LEISTIKOW, Edificios hospitalarios en Europa durante diez siglos, Frankfurt am Main, 1967, pp. 69-70. de enfermos está garantizada por cinco patios (nº 43). En los extremos de cada pabellón se ubican varios espacios reservados para los jefes de sala (no 19), practicantes ( $n^{\circ}$ 18) y auxiliares sanitarios ( $\mathrm{n}^{\mathrm{o}} 17$ y 32) que siguen el modelo hospitalario francés y funcionan como unidades de atención y vigilancia ${ }^{36}$. En la prolongación de los dos pabellones más extremos de cada flanco se localizan cuatro cuerpos salientes que siguen el eje transversal de las salas de hospitalización y alteran, por tanto, la secuencia horizontal de la crujía; en ellos se emplazan una serie de piezas funcionales: las cocinas (no 22) y los comunes (n⿳⺈ 31).

Dos estructuras aditivas que definen sendos patios cuadrangulares de un solo piso con fuentes y lavaderos ( $n^{-}$48) en su centro completan la planta baja del hospital. De usos variados, acogen almacenes de ropa ( $\left.n^{\circ} 45\right)$, comestibles ( $n^{-}$46) y carbón ( $\left.n^{\circ} 47\right)$, así como algunas habitaciones reservadas para los dependientes ( $\left.n^{\circ} 44\right)$. En el espacio comprendido entre estas dos estructuras bajas, que corresponde a un área ajardinada de acceso restringido, se localizan los denominados pozos para las sanguijuelas ( $\left.\mathrm{n}^{\mathrm{0}} 49\right)$, una puerta de servicio que facilita el traslado discreto de los muertos y los depósitos de cadáveres ( $\left.\mathrm{n}^{\mathrm{o}} 50\right)$. Estas últimas dependencias hospitalarias están adosadas al muro de cierre y ocupando la posición más alejada posible de la entrada principal, aunque cerca de la capilla ${ }^{37}$.

\footnotetext{
${ }^{36}$ Sobre el modelo de pabellones desarrollado en Francia a finales del siglo XVIII, véase M. D. FERNÁNDEZ MÉRIDA, "Aproximación a la historia de la arquitectura hospitalaria", Cuadernos de Arte e Iconografía, $\mathrm{n}^{\circ}$ 29, 2006, pp. 72-73 y 88-90.

${ }^{37} \mathrm{Al}$ ilustrado español Valentín de Foronda, difusor en el País Vasco de las ideas que sobre la edificación de hospitales desarrolló la Academia de las Ciencias de París, me voy a referir más adelante. Apuntar simplemente que Foronda era partidario de colocar el depósito de los cadáveres detrás de la capilla, tal como establece Marrón en su proyecto. $\mathrm{Al}$ respecto, véase $\mathrm{V}$. FORONDA, Memorias leídas en la Real Academia de las Ciencias de París sobre la edificación de hospitales, Madrid, 1793 , p. 29.
} 
La última planimetría que forma parte de la prueba de pensado de Balbino Marrón y Ranero describe la compartimentación de la planta alta (Planta principal) del hospital general $^{38}$ (Fig. 4). Su crujía principal, tanto en superficie como en distribución, es prácticamente idéntica a la del piso bajo, variando únicamente en la función prevista para las diferentes salas instaladas. Ocupando una superficie equivalente a la sección central de la planta baja, aparecen delineadas en este piso alto la amplia balaustrada que delimita el balcón de la fachada principal del edificio; la sala de juntas ( $\left.n^{\mathrm{o}} 1\right)$, que abarca tres estancias; las habitaciones del director ( $\mathrm{n}^{\mathrm{o}} 2$ ) y el inspector ( $\left.n^{-} 3\right)$; y el segundo tramo de la escalera principal ( $\left.\mathrm{n}^{\mathrm{o}} 4\right)$. A continuación, en el flanco izquierdo, que corresponde al sector femenino del hospital, se localizan los denominados salones de desahogo para las convalecientes (no 40); el tramo alto de la escalera de servicio y los cuartos de enfermas de distinción ( $\mathrm{n}^{\mathrm{o}} 41$ ). La sala de operaciones de cirugía ( $\mathrm{n}^{\mathrm{o}}$ 38) y dos piezas anexas a ella identificadas como los gabinetes del aparato ( $\mathrm{n}^{\circ} 39$ ) ocupan el extremo de la crujía por ese lado. La compartimentación de estancias y la distribución de funciones se repiten en el flanco derecho reservado a los pacientes masculinos $\left(n^{\circ} 9\right.$, 10, 11 y 12).

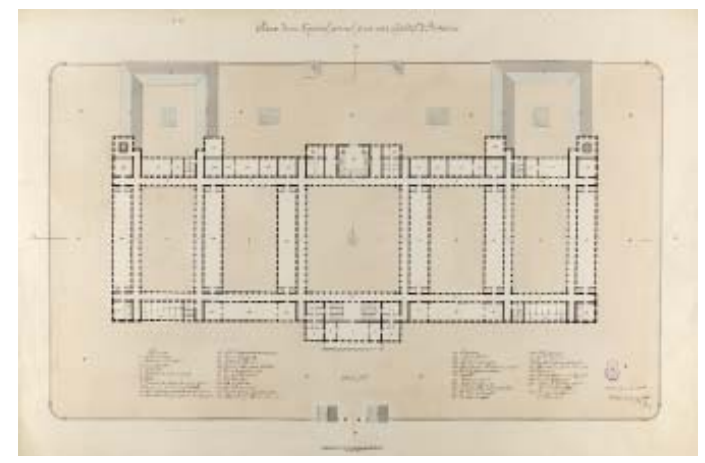

- Fig. 4. Hospital general: planta principal. Proyecto de Balbino Marrón, 1837. Archivo de la Real Academia de Bellas Artes de San Fernando, Gabinete de dibujos, A-2504.

${ }^{38}$ ARABASF, Gabinete de Dibujos, A-2504.
La crujía posterior de esta planta principal del hospital también respeta la estructura y disposición del piso bajo. En el centro se localiza la capilla ( $n^{-0} 27$ ), a la que se accede exclusivamente por las tribunas altas ( $\left.\mathrm{n}^{\mathbf{0}} 28\right)$ que facilitan, a los residentes de la planta, la cómoda asistencia a los actos religiosos. Muy cerca de ella figuran delineadas las habitaciones del administrador ( $\left.n^{\circ} 26\right)$ y el enfermero mayor ( $\left.n^{\circ} 25\right)$. A continuación, en el sector izquierdo de las mujeres, se ubican los denominados cuartos de ropa ( $\left.\mathrm{n}^{\mathrm{o}} 32\right)$, el comedor de las convalecientes ( $\left.\mathrm{n}^{\circ} 35\right)$ y las salas de las presas ( $\mathrm{n}^{\circ}$ 33) y los guardias ( $\left.n^{\circ} 34\right)$, distribución que se repite en el otro lado, en la zona de los hombres (no 17, 20, 18 y 19). Los extremos, en ambos flancos, son ocupados por sendas áreas de baños de vapor (n⿳⺈ 24) y sanitarios (no 23) y más cuartos de ropa (no 32 y 17). Adosados a los lados de este frente posterior del piso alto, aparecen dibujados los tejados de los dos patios cuadrangulares que se sitúan en la planta baja del hospital y los correspondientes al depósito de cadáveres y al cuerpo de guardia (no 7).

Las plantas altas de los seis pabellones que enlazan transversalmente las crujías longitudinales del piso principal ( $\mathrm{n}^{\mathrm{o}} 13$ y 29) presentan una disposición similar a las del piso bajo, tanto en estructura como en compartimentación y funciones, con espacios para los jefes de sala ( $\left.\mathrm{n}^{\mathrm{o}} 16\right)$, practicantes (no 15 y 31) y auxiliares sanitarios ( $\mathrm{n}^{\mathrm{o}} 14$ y 30 ), en consonancia con el modelo francés de pabellones ${ }^{39}$. También incorporan sus respectivas galerías de tránsito ( $\mathrm{n}^{\circ}$ 5). Los cuatro cuerpos salientes que prolongan perpendicularmente los dos pabellones más extremos de cada flanco mantienen las mismas funciones de almacenes y comedores $\left(n^{\circ} 17,21,32\right.$ y 36$)$ o espacios comunitarios $\left(n^{\circ} 22,37\right)$ que prestan en la planta baja, tanto en el sector femenino como en el masculino.

En el hospital general proyectado por Balbino Marrón para la ciudad de Se-

\footnotetext{
${ }^{39}$ Véase la nota $n^{\circ} 36$.
} 
villa se establecen, en resumen, las siguientes áreas: en la fachada principal se localizan un bloque central administrativo, que ocupa los dos pisos; la botica, situada en la planta baja; un doble sector de recreo, femenino y masculino, dispuesto en el piso principal; dos áreas reservadas para los enfermos de distinción, que comprenden ambas plantas y están separadas por razón de sexo; y un doble sector quirúrgico instalado en los extremos de la crujía que también abarca el piso inferior y el superior. El frente posterior del hospital alberga, en torno a la capilla, un área religiosa que ocupa las dos plantas del edificio; dos sectores dedicados a patologías específicas, en el piso bajo; otros dos bloques de servicios, dispuestos en ambas plantas; y un doble sector que se dedica a baños terapéuticos, para hombres y mujeres, instalado en el piso alto. Las dos estructuras cuadrangulares anexas a esta fachada posterior acogen, en su único piso, más bloques de servicios. En los seis pabellones transversales que enlazan con las dos crujías longitudinales se disponen doce salas de enfermos, dos en cada módulo, separadas asimismo por razón de sexo.

Una vez analizados los planos, cabe preguntarse si la arquitectura para la curación y el cuidado imaginada por Marón responde a una concepción moderna o sigue, por el contrario, fórmulas tradicionales. Para responder adecuadamente esta cuestión, hay que contextualizar la propuesta. En este sentido, la historiografía relativa a la arquitectura hospitalaria occidental destaca el largo proceso de evolución tipológica que han sufrido los hospitales. Arranca con los diseños basilicales y claustrales de la Antigüedad y la Edad Media, continúa con las formas cruciformes de los hospitales italianos del Renacimiento y los españoles levantados por los reyes a partir de 1501, y culmina en la segunda mitad del siglo XVIII, cuando el hospital deja de ser considerado un espacio asistencial y se perfila ya, siguiendo criterios higienistas, como lugar específico de atención a los enfermos ${ }^{40}$.

En la época en que Balbino Marrón pergeñó su proyecto, la ciudad burguesa demandaba equipamiento para el ocio, la administración, el comercio y la docencia, pero las instalaciones sanitarias también eran muy valoradas por la sociedad. La definición del hospital moderno del siglo XIX sufre, no obstante, un complejo y dilatado proceso de búsqueda tipológica que se inicia en las décadas finales de la centuria anterior con el debate en torno a la planta y el enclave óptimo para del nuevo HôtelDieu de París, a raíz de su incendio en 1772. Aunque no voy a referirme a los diferentes tipos proyectados para este importante establecimiento sanitario, debo destacar la propuesta de Jean-Baptiste Le Roy y Charles-François $\mathrm{Viel}^{41}$, quienes plantearon la reconstrucción del hospital a partir de un modelo de pabellones de un solo piso dispuestos a derecha e izquierda de un gran patio central siguiendo, probablemente, los principios constructivos de los hospitales ingleses de San Bartolomé de Londres y la base naval de Plymouth ${ }^{42}$. Este prototipo de Le Roy y Viel, algo reformado, eso sí, porque se le aplicaron principios médicos y funcionales y las teorías higienistas de la época que consideraban el aire como el medio más propicio para el contagio de enfermedades, fue convertido por la Academia

${ }^{40}$ Sobre esta cuestión, véase D. LEISTIKOW, Op. cit., pp. 7-10.

${ }^{41}$ El proyecto de Le Roy y Viel data de 1773 pero hasta 1777 no se presentó a la Academia de Ciencias de París, y su publicación, además, se prolongó diez años. $\mathrm{Al}$ respecto, véanse A. BONET CORREA, "El Hospital de Belén en Guadalajara (México) y los edificios de planta estrellada", en Morfología y Ciudad, Barcelona, 1978, pp. 118-119; y M. D. FERNÁNDEZ MÉRIDA, Op. cit., p. 69.

${ }^{42}$ El hospital de San Bartolomé, edificado en 1730, se estructuraba en cuatro bloques independientes dispuestos sobre los lados de un amplio patio. El hospital naval de Plymouth, construido hacia 1764 , incorporaba catorce módulos conectados mediante galerías. Sobre esta cuestión, véanse D. LEISTIKOW, Op. cit., p. 94 y N. PEVSNER, Op. cit., pp. 179-180. 
de las Ciencias de París en el hospital oficial del siglo XIX. La planta paradigmática del denominado sistema de pabellones, que separaba las distintas enfermedades en diferentes salas más o menos aisladas, la dibujó en 1786 Bernard Poyet (Fig. 5), según el programa aprobado por la Academia francesa ${ }^{43}$.



- Fig. 5. Hospital de pabellones. Planta de Bernard Poyet según el programa de la Academia de las Ciencias de París, 1786. Publicada en V. FORONDA, Memorias leídas en la Real Academia de las Ciencias de París sobre la edificación de hospitales, Madrid, 1793.

Pero en 1837, cuando el joven Marrón elaboró su prueba de pensado, no se había aceptado plenamente el modelo. En Francia, de hecho, habrá que esperar a la erección del Hospital Lariboisière de París, proyectado en 1839 y construido entre 1846-1854 por

${ }^{43}$ Con este sistema se lograba que cada pabellón funcionase en realidad como un hospital separado y especializado que compartía los servicios comunes. Al respecto, véase Q. ONASTRA, “Los orígenes del lazareto pabellonario. La arquitectura cuarentenaria en el cambio del Setecientos al Ochocientos", Asclepio. Revista de Historia de la Medicina y de la Ciencia, Vol. LX, no 1, 2008, pp. 238-248.
Gauthier, para que triunfe el sistema de pabellones $^{44}$. Y en España no se adoptará de forma generalizada hasta la segunda mitad de la centuria, a partir, concretamente, del hospital madrileño de la Princesa, edificio proyectado en 1851 por Aníbal Álvarez Bouquel e inaugurado seis años más tarde, que contó con el apoyo explícito de la Comisión de Arquitectura ${ }^{45}$. Aunque las novedades europeas en materia higiénico-sanitaria tuvieron eco en la Academia de San Fernando desde finales del siglo XVIII y los proyectos de edificios hospitalarios fueron promovidos como tema de examen para obtener premios, pensionados y títulos, los diseños de hospitales conservados en el archivo de esta institución correspondientes a la primera mitad del siglo XIX son heterogéneos y presentan un amplio abanico de modelos: claustral, cruciforme, palacial, radial y con pabellones ${ }^{46}$.

Balbino Marrón, no obstante, conocía la utilidad y modernidad del sistema de pabellones hospitalarios. Tal vez aconsejado por Melchor Cano, su maestro y tutor en Sevilla, consultó algún libro de Durand, como Recueil et Parallèle $e^{47}$, texto donde el arquitec-

${ }^{44}$ N. PEVSNER, Op. cit., pp. 182 y 185. Este hospital francés, al igual que ocurre en el proyecto de Balbino Marrón, también presenta el sector administrativo y la capilla enfrentados y separados por un gran patio.

${ }^{45}$ P. NAVASCUÉS PALACIO, Arquitectura y arquitectos madrileños del siglo XIX, Madrid, 1973, p. 107.

${ }^{46}$ M. D. FERNÁNDEZ MÉRIDA, Op. cit., pp. 138-146. La autora recopila una representativa selección de diseños hospitalarios conservados en la Academia en pp.143167. La difusión de los Elementos de Matemáticas de Benito Bails, texto donde el académico de San Fernando copia literalmente el modelo radial hospitalario desarrollado por el francés Antoine Petit en 1774, influye tipológicamente en muchos proyectos del período que se está estudiando, a pesar de que en 1783, año en que Bails publica su libro, ya estaba superado en Francia (al respecto, véase C. SAMBRICIO, Op. cit., pp. 131-140). Sobre la importante labor realizada por los ingenieros militares españoles en materia hospitalaria, véase J. M. MUÑOZ CORBALÁN, “Sanidad, higiene y arquitectura en el siglo XVIII: los ingenieros militares, un eslabón en la política sanitaria y hospitalaria borbónica", Boletín de Arte, nº 11, 1990, pp. 119-136.

${ }^{47}$ J. N. L. DURAND, Recueil et Parallèle des édifices de tout genre anciens et modernes, París, 1800. 
to francés, tras comparar varios ejemplos de hospitales europeos, se decanta por los articulados mediante módulos, o Précis des leçons $d$ 'Architecture, que reproduce, en forma de grabado, un diseño de Poyet para un hospital francés estructurado mediante pabellones en torno a un gran patio central rectangular ${ }^{48}$. Cano, de hecho, conocía y valoraba los textos de Durand porque en su disertación con motivo de su nombramiento como académico de mérito copió pasajes literales del último libro referido del autor francés ${ }^{49}$.

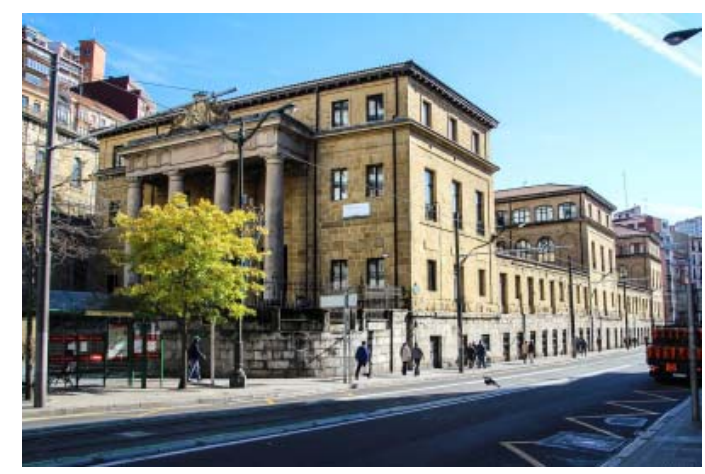

- Fig. 6. Antiguo hospital de Atxuri, hoy Instituto Emilio Campuzano. Bilbao.

Existe, con todo, un singular edificio construido en el reinado de Fernando VII en el que se aplica por primera vez en España el modelo sanitario de pabellones: el hospital bilbaíno de Atxuri (Fig. 6), hoy Instituto Emilio Campuzano. Sus planos fueron concebidos por Gabriel Benito de Orbegozo en 1818, bajo la influencia de las ideas de Valentín de Foronda, difusor, en el País Vasco,

${ }^{48}$ ÍDEM, Précis des leçons d'Architecture, vol. II, París, 1802, lámina 18.

${ }^{49}$ Sobre esta cuestión, véase M. D. ANTIGÜEDAD DEL CASTILLO-OLIVARES, Op. cit., pp. 424-426. Es muy posible, por otra parte, que Cano, a la hora de buscar fuentes de inspiración para el diseño de los nuevos pabellones previstos en el mercado sevillano de la Feria, siguiese, parcialmente, por lo menos, los modelos de los mercados incluidos por Durand en Précis des leçons d'Architecture (al respecto, véase A. FERNÁNDEZ GONZÁLEZ, "Un emblemático edificio de Sevilla: historia constructiva, planos y proyectos del histórico mercado de la Feria", Boletín del Museo e Instituto Camón Aznar, $\mathrm{n}^{\mathrm{o}}$ 111, 2013, p. 73). del ideario ilustrado sobre la edificación de hospitales que desarrolló la Real Academia de las Ciencias de París ${ }^{50}$. Balbino Marrón, por fuerza, tenía que conocer este complejo sanitario, pues precisamente en Bilbao, de la mano de Juan Bautista Belaunzarán, había iniciado su formación como arquitecto ${ }^{51}$. Se trata de un edificio que tiene una planta en forma de peine -como la proyectada por Marón- y desarrolla un sistema estructural de tres pabellones integrados, no aislados, que Orbegozo liga a galerías de comunicación y a dos grandes patios centrales de ventilación que permitían separar los enfermos por razón de su sexo ${ }^{52}$. El modelo estructural, en esencia, es muy similar al planteado por el futuro arquitecto hispalense en su prueba de pensado, aunque el proyecto del joven Marón, claro está, es un ejercicio de examen no supeditado a la realidad constructiva, y por este motivo, precisamente, es mucho más monumental y compacto, tiene un mayor número de patios y pabellones, más ejes de circulación y rotundas crujías de enlace en vez de sencillos corredores ${ }^{53}$. Este sistema de pabellones integrados utilizado por Orbegozo y Marrón, que en las décadas centrales del siglo XIX encarnaba la arquitectura hos-

${ }^{50}$ La planta correspondiente al proyecto de hospital delineado por Bernard Poyet según el programa de la Academia de las Ciencias de París (Fig. 5) se reproduce en V. FORONDA, Op. cit., p. 81. Sobre la influencia de Valentín de Foronda en el hospital construido por Gabriel Benito de Orbegozo, véase K. M. BARAÑANO, J. GONZÁLEZ DE DURANA y J. JUARISTI, Op. cit., p. 167. También resulta muy instructivo C. SAMBRICIO, Op. cit., pp. 52 y 129-145.

${ }^{51}$ ARABASF, Comisión de Arquitectura, Expediente 2-4-6, s. fol.

${ }^{52}$ Sobre este edificio hospitalario, véanse J. GONZÁLEZ DE DURANA, "Antiguo hospital de AchuriBilbao", en Monumentos de Vizcaya, vol. IV, Bilbao, 1987, pp. 283-291 y P. NAVASCUÉS PALACIO, Arquitectura española 1808-1914. “Summa Artis", vol. XXXV, Madrid, 1996, p. 153.

${ }^{53}$ La Academia de San Fernando, en el tema de los hospitales, como explica M. D. FERNÁNDEZ MÉRIDA, Op. cit., p. 167, "mostraba la ambivalencia con la que la propia arquitectura se iba desarrollando entre las formas generadas por la necesidad, programa o economía y las ligadas a las fuentes figurativas clásicas". 
pitalaria más avanzada ${ }^{54}$, también fue empleado para renovar el prestigioso hospital de St. Thomas de Londres (Fig. 7).

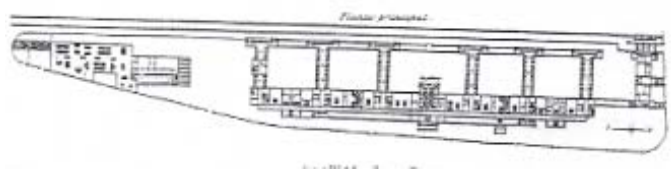

- Fig. 7. Planta del hospital londinense de St. Thomas. Henry Currey, 1871. Publicada en E. LABAIG, Hospitales civiles y militares, Madrid, 1883.

El proceso constructivo del hospital general es explicado en el informe facultativo, donde refiere Balbino Marrón, en primer lugar, la fase inicial de desmontado y explanado del terreno para hablar a continuación del trazado de los ejes maestros del edificio. Mediante equidistancias y perpendiculares se tendrían que establecer las dimensiones de las crujías y galerías y los espesores de los $\operatorname{muros}^{55}$. La apertura de zanjas hasta la profundidad adecuada a la naturaleza del terreno y la elevación de los muros constituyen las fases siguientes de los trabajos de edificación. Una vez realizadas estas labores constructivas, según explica el futuro arquitecto municipal y provincial de Sevilla, “enseguida mandaría rellenar y macizar los cimientos, de cal y canto, obra de mampostería irregular, pero bien enlazada hasta cerca del nivel del terreno, a fin de completar este con las losas de elección sobre que formaría el replanteo de los muros y sus gruesos determinados, señalando la magnitud y dimensiones de los vanos, puertas y arcos que sirven de ingresos y comunicaciones para de esta suerte pasar enseguida a la colocación del zócalo general de sillería" ${ }^{\prime 56}$.

${ }^{54}$ A finales del ochocientos, no obstante, se generalizará en Europa la moda de pabellones completamente aislados. Al respecto, véase A. BONET CORREA, Op. cit., p. 119.

${ }^{55}$ ARABASF, Comisión de Arquitectura, Expediente 2-4-6, informe facultativo, fols. 5 r. $-5 \mathrm{v}$.

${ }^{56}$ Ibídem, fol. 6 r.
El suelo de la planta baja del hospital imaginado por el joven Marrón debía elevarse cuatro pies a fin de evitar humedades ${ }^{57}$. Aunque los dinteles, fajas, cornisas, ángulos y arcadas de las fachadas se tendrían que fabricar de cantería, la mampostería constituye el principal material constructivo ${ }^{58}$. Al final del informe facultativo se detalla el presupuesto de la obra, que alcanza los 8.689.384 reales de vellón ${ }^{59}$.

La comisión de evaluación se reúne el 13 de agosto de $1837 \mathrm{y}$, después de enjuiciar favorablemente los planos y el informe, fija el siguiente ejercicio de examen una semana más tarde. Esta segunda fase de la oposición, denominada prueba de repente, tenía un carácter más práctico ${ }^{60}$. Del Libro de asuntos para arquitectos, texto que establecía un programa de ciento cincuenta ejercicios a realizar "en tintas y manchados" ${ }^{61}$, al joven Marrón le tocaron en suerte el tema noventa y tres, que consistía en el diseño de una "escalera principal de un palacio con galería interior que la rodee y puertas para varios cuartos"; el número cuatro del programa, que se refería a la planta, trazado y sección de "la fachada de un palacio real, cuya mayor extensión no exceda a mil pies castellanos ni menos de quinientos, y su elevación comprenderá piso bajo, principal y un entresuelo, todo bajo la cornisa general y particular de la misma"; y el tema sesenta y dos, que fijaba la delineación en planta, alzado y cor-

\footnotetext{
${ }^{57}$ Los suelos de los pabellones de los enfermos llevarían "entablados a manera de cubierta de barco para que así puedan barrerse del mismo modo que se hacen aquellas"; los de las habitaciones, un pavimento de ladrillos; y de losas serían los suelos de las galerías, pórticos y zaguanes (Ibídem, fol. 7 r.). Evitar las perniciosas humedades en la edificación de hospitales es un principo básico constructivo ya apuntado por B. BAILS, Op. cit., p. 857.

${ }^{58}$ ARABASF, Comisión de Arquitectura, Expediente 2-4-6, informe facultativo, fol. $6 \mathrm{v}$. Todas las escaleras se proyectan de madera, salvo la pétrea escalinata del frente principal (Ibídem., fols. 7 r.-7 v.).

${ }^{59}$ Ibídem fols. 8 r. -9 v.

${ }^{60} \mathrm{Al}$ respecto, véase J. E. GARCÍA MELERO, “Arquitectura y Burocracia...", pp. 283-348.

${ }^{61}$ M. R. SANTAMARÍA ALMOLDA, Op. cit., p. 221.
} 
te de "un arca principal de repartimiento de aguas para todas las fuentes públicas y particulares de una población tan grande como Madrid, adornada con la arquitectura que corresponde, en el supuesto de hacer monumento público dentro de la población" ${ }^{62}$. Balbino Marrón se decantó finalmente por el tema número cuatro, el correspondiente a la fachada de un palacio real ${ }^{63}$.

Además de concurrir a las pruebas de pensado y de repente, el futuro arquitecto hispalense tuvo que superar un examen oral el 6 de septiembre ante el tribunal constituido por Juan Miguel de Ynclán, Custodio Teodoro Moreno, Tiburcio Pérez y Miguel de Loredo. En el acta de evalución redactada por Marcial Antonio López, que ejercía las funciones de secretario de la comisión, consta que los académicos "examinaron las obras de pensado y de repente que había ejecutado, mandándole entrar para que las explicase, haciéndole después algunas objeciones, a las que contestó". A continuación se le preguntó acerca de los números, de las ecuaciones de diversos grados, de las proporciones, las líneas y sus especies; y también sobre ángulos, triángulos y cuadriláte-

${ }^{62}$ ARABASF, Comisión de Arquitectura, Expediente 2-4-6, s. fol.

${ }^{63}$ Sobre este diseño, véase J. LABORDA YNEVA, Op. cit., p. 313. ros y sobre el modo de hallar las superficies. De las distintas fases constructivas de los edificios y los procedimientos para desalojar de agua los terrenos tampoco se olvidó el tribunal. Los académicos lo interrogaron asimismo sobre los replanteos, la elección y colocación de las maderas, los requisitos necesarios para la buena construcción y sobre los arcos y sus diferencias. Concluido el examen, "se mandó retirar al interesado y acto continuo se procedió a la votación secreta de la que resultó aprobado por tres votos a favor y uno en contra, acordando la junta se haga presente a la Academia, por si se digna conformar con este dictamen" ${ }^{\prime 64}$.

Balbino Marrón, meses más tarde, consigue la plaza de arquitecto municipal de Jerez de la Frontera ${ }^{65}$, y aunque en 1845 realiza el levantamiento planimétrico del desaparecido hospital sevillano del Espíritu Santo ${ }^{66}$ y al año siguiente, ya como arquitecto titular del Ayuntamiento hispalense, reforma el antiguo hospital del Cardenal para adaptarlo a lo que fuera el asilo de mendicidad de San Fernando ${ }^{67}$, nunca tendrá ocasión de materializar sus modernas ideas sobre arquitectura hospitalaria.
${ }^{64}$ ARABASF, Comisión de Arquitectura, Expediente 2-4-6, s. fol.

${ }^{65}$ J. M. SUÁREZ GARMENDIA, Arquitectura y urbanismo..., p. 99.

${ }^{66} \mathrm{Al}$ respecto, véase $\mathrm{F}$. J. MOCLOVA GONZÁLEZ, "Aproximación a la arquitectura hospitalaria en Sevilla durante la Edad Moderna: estudio de los planos y edificios de los hospitales del Amor de Dios y Espíritu Santo", Archivo Hispalense, no 285-287, 2011, p. 432.

${ }^{67}$ Sobre este edificio, véase M. C. GIMÉNEZ MUÑOZ, El asilo de mendicidad de San Fernando (1846-1900), Sevilla, 2006 